\title{
Self-induced liquid crystal q-plate by photoelectric interface activation
}

\author{
Nina Kravets, ${ }^{1}$ Nina Podoliak, ${ }^{2}$ Malgosia Kaczmarek, ${ }^{2}$ and Etienne Brasselet ${ }^{1, \text { a) }}$ \\ 1) Univ. Bordeaux, CNRS, Laboratoire Ondes et Matière d'Aquitaine, F-33400 Talence France \\ ${ }^{2)}$ Physics and Astronomy, University of Southampton, Southampton SO17 1BJ UK
}

(Dated: 14 December 2018)

\begin{abstract}
Here we report on the experimental demonstration that highly efficient self-induced spin-orbit optical vortex generation can be achieved by using standard liquid crystal materials and surface treatment agents. This is done by revisiting recent attempt by Zolot'ko and coworkers to produce self-induced liquid crystal vortex plates using dc electric field [Mol. Cryst. Liq. Cryst. 637, 47 (2016)] that remains, so far, limited to moderate efficiencies. The phenomenon is the result of the self-back-action of light arising from the spontaneous creation of a liquid crystal topological defect. These results demonstrate photo-electric interface activation as a candidate towards the development of a self-adapted spin-orbit photonic toolbox, thus enabling agile management of the orbital angular momentum of light.
\end{abstract}

Since the early days of a research field dedicated to the study and use of the orbital angular momentum of light $^{1}$, numerous strategies have been developed to manipulate the spatial degrees of freedom of a light field. In the paraxial regime, the description of the optical orbital angular momentum is commonly associated with wavefront shaping according to a pure phase factor $\exp (i \ell \phi)$ where $\ell$ integer is the topological charge of the phase singularity and $\phi$ is the polar angle in the plane transverse to the light's propagation direction. The development of optical elements imparting helical phase profiles to light beams is therefore a key technological requirement.

Two kinds of optical phase shapers have been designed for such task: scalar and vectorial ones, which respectively rely on space-variant dynamic phase (case 1) and geometric phase (case 2). A prototypical example of the case 1 refers to refractive spiral phase plates - threedimensional optically isotropic objects whose thickness varies linearly with the azimuthal coordinate. Since the first demonstration in $1992,{ }^{2}$ nanofabrication technologies have emerged that now allow creating optically perfect spiral phase plates, also endowed with additional structural information related to the radial degree of freedom of light ${ }^{3}$. The case 2 is based on the spinorbit interaction of light and exploits the opposite phase shifts imparted on the two orthogonal circular polarization states under rotations of the spatial coordinates of optically anisotropic media ${ }^{4}$. The first demonstration dates back $2002^{5}$ and since then robust technologies have been developed, based on various solid-state materials that are either naturally or artificially birefringent (e.g., dielectrics, metals, glasses and liquid crystal polymers). Remarkably, both dynamic and geometric phase properties can be merged into 'geometrodynamic' phase optical elements ${ }^{6}$.

Still, despite an indisputable level of maturity that has been reached, an agile toolbox to manage the orbital angular momentum of light temporally, spatially and spectrally remains as a significant challenge.

a)Electronic mail: etienne.brasselet@u-bordeaux.fr
Tunable and reconfigurable features obviously require materials whose optical properties can be modified under external stimuli. Liquid crystals are promising spinorbit candidates owing to their sensitivity to electrical and magnetic fields. They have been successfully applied in spectrally tunable optical vortex generators with ${ }^{7}$ or without $^{8}$ surface patterning of the liquid crystal orientation. Adaptive multiple vortex masks have also been achieved $^{9,10}$. Moreover, combining spectral and spatial control capabilities into a single device provides multispectral vortex modulation, as reported in Ref. 11. Based on such prospects, the study of optical elements, endowed with self-controlled spin-orbit photonic functionalities, is an important topic to study for its scientific and technological potential. Over the past few years, various situations showed self-induced liquid crystal topological reorientation taking place under all-optical ${ }^{12,13}$, photoassisted ac-electrical $^{14}$, photo-assisted dc-electrical ${ }^{15}$ or photo-thermal processes ${ }^{16}$. Finally, a recent work reporting on liquid crystal defects generated by photoactivated interface between iron doped lithium niobate and liquid crystals $^{17}$ suggests an alternative approach worth being explored. All liquid crystal orientational patterns obtained correspond to the self-written version of a so-called q-plate with topological charge $q=1,{ }^{18}$ enabling a change of orbital angular momentum associated with $\delta \ell= \pm 2$ depending on the incident photon helicity $\sigma= \pm 1$.

Importantly, two key figures of merit of such vortex generators are the purity (i.e. the fraction of the output power that is associated with an orbital angular momentum change) and the efficiency (i.e., the transmittance of the generated vortex state). To date, only the photo-assisted ac-electrical option ${ }^{14}$ has demonstrated self-induced vortex generation with high purity, while the efficiency remains inherently limited to the use of absorbing photoconductive crystals ${ }^{19}$. In contrast, the photoassisted dc-electrical option ${ }^{15}$ is based on the photoelectric interfacial effect between a thin polymer layer, coated on a glass substrate with a transparent electrode, and a liquid crystal thin film ${ }^{20}$. Therefore, this option has the potential for being simultaneously pure and 


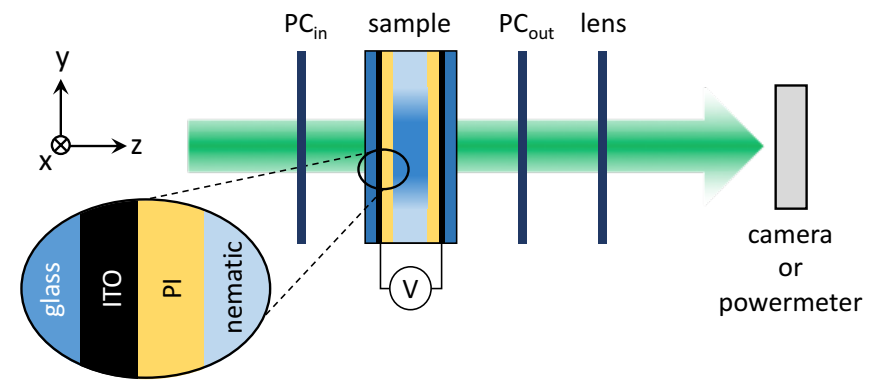

FIG. 1. Sketch of the experimental setup. $\mathrm{PC}_{\text {in/out }}$ input/output polarization controllers. The lens is placed to image either the sample plane (using $75 \mathrm{~mm}$ focal length) or the far-field via optical Fourier transform (using $150 \mathrm{~mm}$ focal length) onto the camera.

efficient ${ }^{21}$, despite reported record efficiencies of the order of $\sim 30 \% .{ }^{22}$ Here, we revisit previous attempts ${ }^{15,22}$ and we experimentally demonstrate that highly efficient (>90\%) self-induced spin-orbit optical vortex generation can be achieved.

In our experiments we use a $17 \mu \mathrm{m}$-thick layer of a nematic liquid crystal having negative dielectric anisotropy at low frequencies. Namely, the liquid crystal mixture MLC-6608 (Merck Japan) that is characterized by a dielectric anisotropy $\delta \epsilon=\epsilon_{\|}-\epsilon_{\perp}=-4.2$ at $1 \mathrm{kHz}$ frequency, a birefringence $\delta n=n_{\|}-n_{\perp}=0.08$ at $589 \mathrm{~nm}$ wavelength, and a bend elastic constant $K_{3}=18,1 \mathrm{pN}$, all given at $20^{\circ} \mathrm{C}$ temperature. The subscripts $(\|, \perp)$ refer to direction parallel and perpendicular to the director, i.e., the headless unit vector that defines the average molecular orientation. The nematic film is sandwiched between two transparent glass substrates provided with indium tin oxide (ITO) electrodes on which is spin-coated a thin layer of a standard polyimide (PI) alignment material (SE-1211, from Nissan) that provides perpendicular boundary conditions for the director field. A sketch of the sample and of the setup is shown in Fig. 1, where the laser beam is a continuous-wave fundamental Gaussian beam at $532 \mathrm{~nm}$ wavelength.

Highly efficient vortex generation implies that the sample behaves everywhere as a half-wave plate. Namely, the birefringent phase retardation, $\Delta$, is expected to satisfy the condition $\Delta=\pi .^{5}$ It corresponds to full polarization conversion of an incident circular polarization state with helicity $\sigma$ into the orthogonal polarization state with helicity $-\sigma$. The first task is therefore to identify the required dc voltage. This can be done by preparing a circularly polarized incident beam with helicity $\sigma$. In our case it had a waist radius of $1.3 \mathrm{~mm}$ and power of $0.25 \mathrm{~mW}$. Then, we evaluate the ratio

$$
\eta=\frac{P_{-\sigma}}{P_{-\sigma}+P_{+\sigma}},
$$

where $P_{ \pm \sigma}$ is the power of the circularly polarized component of the output beam with helicity $\pm \sigma$. The results are reported in Fig. 2 where the dependence of $\eta$ as a function of the applied voltage $V$ is shown for both $\mathrm{dc}$ and ac situations for comparison. In the ac case, the liquid crystal reorientation is detected above $2.2 \mathrm{~V}$ in agreement with the electrical reorientation threshold voltage given by $V_{\mathrm{th}}=\pi \sqrt{K_{3} /\left(\epsilon_{0} \mid \delta \epsilon\right) \mid}=2.19 \mathrm{~V}$ and the sought-after half-wave retardation condition corresponds to $V_{\pi}=2.63 \mathrm{~V}$. In the dc case, the whole set of measurements is performed over the duration of three minutes, hence minimizing the back-action of light at the $\mathrm{PI} /$ nematic interface, connected to the positive pole of the electrical power supply ${ }^{20}$, as confirmed by the results in Fig. 3(a). The fact that reorientation takes place above an applied voltage that is slightly larger than for the ac case, and that $V_{\pi}^{\text {dc }} \gtrsim V_{\pi}^{\text {ac }}$ in the framework of our transient measurement protocol, can both be understood by partial charge migration and also by the dispersion of the dielectric anisotropy.

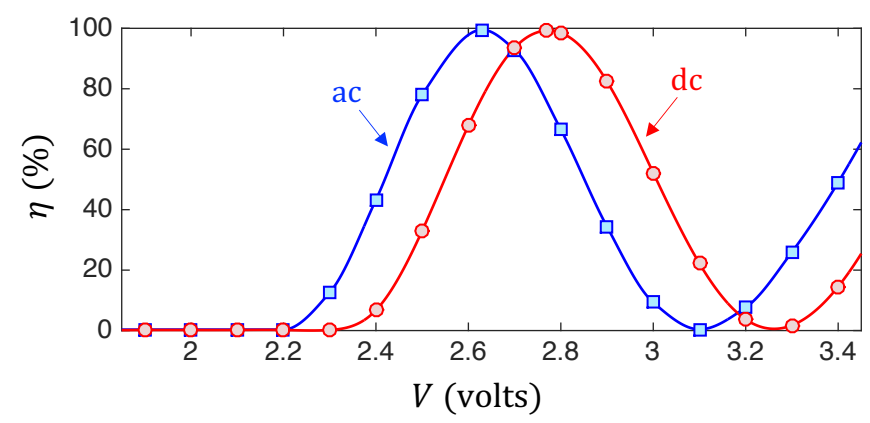

FIG. 2. Purity of the polarization conversion as a function of the applied voltage in the ac (at $1 \mathrm{kHz}$ frequency, using a square waveform) and dc condition. Markers: experimental data. Solid curves: spline adjustment intended to guide the eye. The half-wave retardation condition is respectively fulfilled for $V_{\pi}^{\text {ac }}=2.63 \mathrm{~V}$ and $V_{\pi}^{\text {dc }}=2.77 \mathrm{~V}$. Incident beam parameters: waist radius is $1.3 \mathrm{~mm}$ and total power is $0.25 \mathrm{~mW}$. Here we recall that the transient measurements protocol ensures that screening effects do not play a significant role in the DC case, as discussed in details in the text.

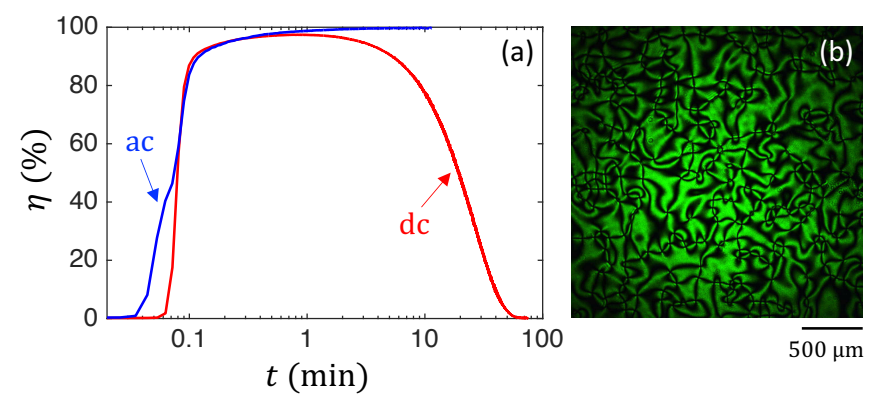

FIG. 3. (a) Polarization conversion dynamics at $V=V_{\pi}$ for ac (blue curve) and dc (red curve) situations. Incident beam parameters: waist radius is $1.3 \mathrm{~mm}$ and total power is $0.25 \mathrm{~mW}$. (b) Typical random network of umbilical defects with topological charge \pm 1 imaged between crossed linear polarizers $30 \mathrm{~s}$ after the voltage is switched on at time $t=0$. 
The second step consists to apply the constant voltage $V=V_{\pi}$ to the sample. In the dc case, charged impurities in the bulk of the nematic migrate to the PI/nematic interfaces in such a way that the external electric field is eventually screened sufficiently to ensure that the effective applied voltage is smaller than $V_{\mathrm{th}}$. This is illustrated in Fig. 3(a) where the long-term dynamics of the polarization conversion is displayed. Indeed, after a few minutes of irradiation $\eta$-hence the liquid crystal reorientation amplitude - has no longer a monotonous increase and eventually decays to zero, in stark contrast to the ac case where $\eta$ asymptotically converges to unity. Note that, when reoriented, the liquid crystal film exhibits in both cases a random collection of nonsingular topological defects called umbilics ${ }^{23,24}$ with topological charge \pm 1 , as illustrated in Fig. 3(b) where the sample is imaged between crossed linear polarizers.

Self-induced, on-axis, umbilic is then obtained in the dc case by local photo-electric effect, which releases the screening. In turn, this activates the local reorientation of the liquid crystal, noting that, despite its photoelectric origin, the applied torque acting on the director is of electrical nature. ${ }^{25}$ Since $V=V_{\pi}$, one can expect full photodescreening to have the potential to create highly efficient q-plates. This implies an appropriate combination of incident power, beam waist, wavelength and materials. In the present case, we used a moderately focused incident

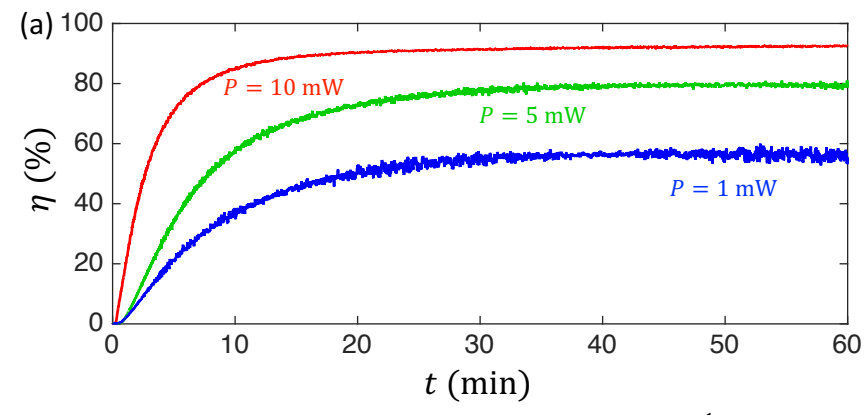

(b)

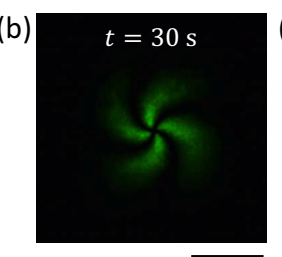

$\overline{250 \mu \mathrm{m}}$
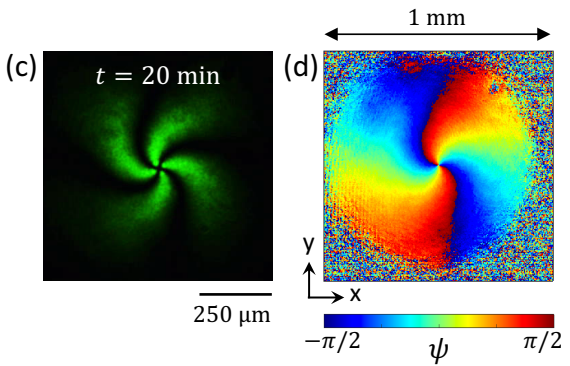

FIG. 4. (a) Dynamics of the spin-orbit optical vortex generation purity after the sample is electrically screened under dc applied voltage $V=V_{\pi}$ for an incident beam waist radius $w=260 \mu \mathrm{m}$ and total power $P=1,5$ and $10 \mathrm{~mW}$. The created localized umbilical defect resulting for the photodescreening process observed between crossed linear polarizers is shown at $30 \mathrm{~s} \mathrm{(b)}$ and $20 \mathrm{~min}$ (c) after the beam is switched on. The orientational map for the director field in the plane of the sample, $\psi(x, y)$, is shown in panel (d).

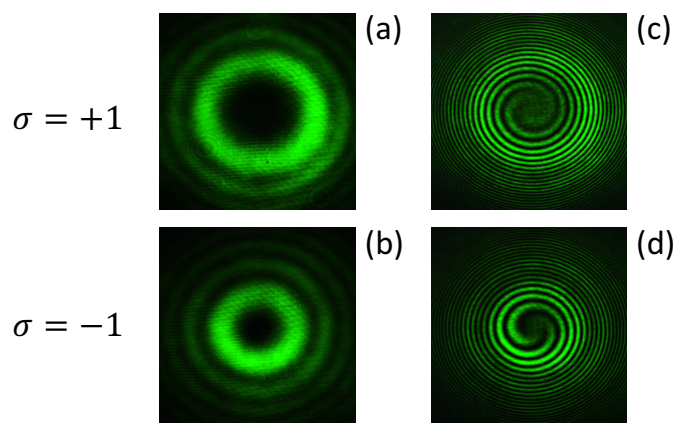

FIG. 5. (a,b) Total far-field intensity profiles of the selfinduced steady-state optical vortex beams generated at $V=$ $V_{\pi}$ in the dc case under total incident power $P=10 \mathrm{~mW}$, incident beam waist radius $w=260 \mu \mathrm{m}$, and incident helicity $\sigma= \pm 1$. (c,d) Spiraling interference patterns obtained by superposing the output beam with a coherent coaxial Gaussian reference beam.

laser beam with waist radius $w=260 \mu \mathrm{m}$ in the liquid crystal layer. Photo-descreening dynamics as a function of the incident beam power, $P$, is shown in Fig. 4(a) where the dynamics of $\eta$ is presented for $P=1,5$ and $10 \mathrm{~mW}$. Steady-state purity as high as $\eta>90 \%$ is obtained for $P=10 \mathrm{~mW}$ and this corresponds to the purity of the spin-orbit vortex generation process. Indeed, the reoriented structure is a localized umbilical defect with topological charge +1 , whose retardance increases with time to reach $\Delta \simeq \pi$ at steady-state, as qualitatively illustrated when imaging the sample between crossed linear polarizers at various stages of the dynamics, as shown in Fig. 4(b) and 4(c). Quantitatively, the effective director structure at steady-state is assessed by spatially resolved polarimetry, see Fig. 4(d) where the map of the in-plane director orientation angle, $\psi$, is displayed. As discussed in previous works, the swirled structure of the umbilic (whose handedness is random) is inherent to the elastic anisotropy of nematic liquid crystals ${ }^{24,26}$.

Self-induced optical vortex generation itself is illustrated in Figs. 5(a) and 5(b) where the total output farfield intensity patterns are shown for both incident helicities $\sigma= \pm 1$. The characteristic doughnut profile associated with a vortex beam is observed and the helicitydependent topological charge $\ell=2 \sigma$ of the vortex is inferred from the interference pattern resulting from the coherent superposition of a Gaussian shaped reference beam, see Figs. 5(c) and 5(d). Indeed, two-arm spiraling patterns, with opposite handedness, are observed for $\sigma= \pm 1$. Note that the distinct radii of the annular intensity profiles are the signature of the above mentioned swirled nature of the localized photo-induced q-plates that act as aspheric axisymmetric vortex lenses. They are best represented by a complex transmittance phase mask of the form $\tau=\exp [2 i \sigma(\phi+f(r))]$, where the real function $f(r)$, with $r$ the distance from the defect core, refers to the deviation of the director orientation with respect to a purely radial structuring given by $\psi=\phi$. 


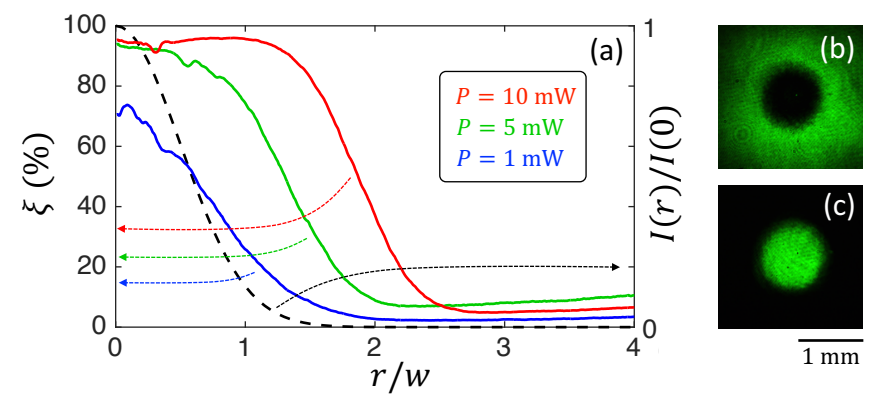

FIG. 6. (a) Colored curves refer to the radial dependence of the azimuth average polarization conversion purity, $\xi$, measured at steady-state for the three experimental situations reported in Fig. 4(a). Dashed curve refers to the normalized Gaussian intensity profile of the writing beam with waist radius $w=260 \mu \mathrm{m}$. Panels (b) and (c): intensity distribution in the plane of the sample of the output circularly polarized components with helicity $+\sigma$ and $-\sigma$, respectively, for $P=10 \mathrm{~mW}$, when collimating transiently the writing light field into a Gaussian beam with waist radius of $1.3 \mathrm{~mm}$.

Observed high efficiency implies a spatial extent of the self-induced q-plate, associated with birefringent phase retardation $\Delta \simeq \pi$, larger than the incident beam waist diameter. It can be experimentally verified by retrieving the radial dependence of the polarization conversion purity. This is done by evaluating, in the sample plane, the ratio

$$
\xi(r)=\frac{\left\langle I_{-\sigma}\right\rangle(r)}{\left\langle I_{-\sigma}\right\rangle(r)+\left\langle I_{+\sigma}\right\rangle(r)},
$$

where $\left\langle I_{ \pm \sigma}\right\rangle(r)=\frac{1}{2 \pi} \int_{0}^{2 \pi} I_{ \pm \sigma}(r, \phi) d \phi$ is the azimuth average radial intensity profile of the output circularly polarized component with helicity $\pm \sigma$. Experimentally, it is realized by modifying transiently the focusing condition of the laser beam, namely, by increasing the beam waist more than five times. This allows probing the sample without affecting the steady-state associated with the focused writing beam. The results that correspond to the steady-state situations, presented in Fig. 4(a), are shown in Fig. 6. We find $\xi(r)>90 \%$ up to $r \simeq 1.3 w$ for $P=10 \mathrm{~mW}$, which highlights the uniformity of the selfinduced q-plate retardance. Finally, regarding relaxation times of the generated space-variant anisotropic optical element, we note that our system has an elastic relaxation time of the order of $1 \mathrm{~s}$ whereas the characteristic time associated with spatial distribution of the electric charges is $>10 \mathrm{~min}$.

Summarizing, the obtained record value for the purity of optical vortex generation process has demonstrated the potential of photoelectric activation of liquid crystal/polymer interfaces as a promising route to develop a self-adapted spin-orbit photonic toolbox for agile management of the orbital angular momentum of light, based on the combined action of light and a dc electrical stimuli. These results, therefore, extend the previous use of liquid crystal light valves based on pho- toconductive crystals to usual polymer-coated transparent glass substrates. Achieved performances can be improved further if air/glass interfaces are properly treated by anti-reflection coatings since Fresnel reflections obviously limit the efficiency.

${ }^{1}$ L. Allen, M. W. Beijersbergen, R. J. C. Spreeuw, and J. Woerdman, Orbital angular momentum of light and the transformation of laguerre-gaussian laser modes, Phys. Rev. A 45, 8185 (1992).

${ }^{2}$ S. Khonina, V. Kotlyar, M. Shinkaryev, V. Soifer, and G. Uspleniev, The phase rotor filter, J. Mod. Opt. 39, 1147-1154 (1992).

${ }^{3}$ G. Ruffato, M. Massari, and F. Romanato, Generation of highorder laguerre-gaussian modes by means of spiral phase plates, Opt. Lett. 39, 5094-5097 (2014).

${ }^{4}$ K. Y. Bliokh, F. J. Rodriguez-Fortuno, F. Nori, and A. V. Zayats, Spin-orbit interactions of light, Nature Photon. 9, 796-808 (2015).

${ }^{5}$ G. Biener, A. Niv, V. Kleiner, and E. Hasman, Formation of helical beams by use of pancharatnam-berry phase optical elements, Opt. Lett. 27, 1875-1877 (2002).

${ }^{6}$ R. C. Devlin, A. Ambrosio, N. A. Rubin, J. B. Mueller, and F. Capasso, Arbitrary spin-to-orbital angular momentum conversion of light, Science 358, 896-901 (2017).

${ }^{7}$ B. Piccirillo, V. D'Ambrosio, S. Slussarenko, L. Marrucci, and E. Santamato, Photon spin-to-orbital angular momentum conversion via an electrically tunable q plate, Applied Physics Letters 97, 241104 (2010).

${ }^{8}$ E. Brasselet, Tunable high-resolution macroscopic selfengineered geometric phase optical elements, Phys. Rev. Lett. 121, 033901 (2018).

${ }^{9}$ R. Barboza, U. Bortolozzo, G. Assanto, E. Vidal-Henriquez, M. G. Clerc, and S. Residori, Harnessing optical vortex lattices in nematic liquid crystals, Phys. Rev. Lett. 111, 093902 (2013).

${ }^{10} \mathrm{~A}$. Aleksanyan, N. Kravets, and E. Brasselet, Multiple-star system adaptive vortex coronagraphy using a liquid crystal light valve, Phys. Rev. Lett. 118, 203902 (2017).

${ }^{11}$ M. G. Nassiri and E. Brasselet, Multispectral management of the photon orbital angular momentum, Phys. Rev. Lett. 121, 213901 (2018).

${ }^{12}$ E. Brasselet, Singular optical manipulation of birefringent elastic media using nonsingular beams, Optics letters 34, 3229-3231 (2009).

${ }^{13}$ M. E. Ketara and E. Brasselet, Self-induced nonlinear spin-orbit interaction of light in liquid crystals, Opt. Lett. 37, 602-604 (2012).

${ }^{14}$ R. Barboza, U. Bortolozzo, G. Assanto, E. Vidal-Henriquez, M. G. Clerc, and S. Residori, Vortex induction via anisotropy stabilized light-matter interaction, Phys. Rev. Lett. 109, 143901 (2012).

${ }^{15}$ I. A. Budagovsky, A. S. Zolot'ko, M. P. Smayev, and S. A. Shvetsov, Formation of the light beam with wavefront screw dislocation at the photorefractive effect in nematic liquid crystal, Bull. Lebedev Phys. Inst. 42, 319-322 (2015).

${ }^{16}$ I. A. Budagovsky, A. S. Zolot'ko, D. L. Korshunov, M. P. Smayev, S. A. Shvetsov, and M. Barnik, Generation of spiral dislocation of wave front in absorbing nematic liquid crystal, Opt. Spectrosc. 119, 280-285 (2015).

${ }^{17}$ A. Habibpourmoghadam, L. Jiao, V. Reshetnyak, D. R. Evans, and A. Lorenz, Optical manipulation and defect creation in a liquid crystal on a photoresponsive surface, Phys. Rev. E 96, 022701 (2017).

${ }^{18}$ L. Marrucci, C. Manzo, and D. Paparo, Optical spin-to-orbital angular momentum conversion in inhomogeneous anisotropic media, Phys. Rev. Lett. 96, 163905 (2006).

${ }^{19}$ P. Aubourg, J. P. Huignard, M. Hareng, and R. A. Mullen, Liquid crystal light valve using bulk monocrystalline $\mathrm{Bi}_{12} \mathrm{SiO}_{20}$ as the photoconductive material, Appl. Opt. 21, 3706-3712 (1982).

${ }^{20} \mathrm{P}$. Pagliusi and G. Cipparrone, Extremely sensitive light-induced reorientation in nondoped nematic liquid crystal cells due to pho- 
toelectric activation of the interface, J. Appl. Phys. 93, 91169122 (2003).

${ }^{21}$ I. A. Budagovsky, A. S. Zolot'ko, and S. A. Shvetsov, Formation of the light beam with wavefront screw dislocation at the photorefractive effect in nematic liquid crystal, Bull. Lebedev Phys. Inst. 43, 340-344 (2016).

${ }^{22}$ I. A. Budagovsky, S. A. Shvetsov, and A. S. Zolot'ko, Optical vortex generation in homeotropic nlcs in the presence of dc electric field, Mol. Cryst. Liq. Cryst. 637, 47-52 (2016).

${ }^{23}$ A. Rapini, L. Léger, and A. Martinet, Umbilics: Static and $d y-$ namic properties, Le Journal de Physique Colloques 36, 189-196 (1975).

${ }^{24}$ M. G. Clerc, E. Vidal-Henriquez, J. D. Davila, and M. Kowalczyk, Symmetry breaking of nematic umbilical defects through an amplitude equation, Phys. Rev. E 90, 012507 (2014).

${ }^{25}$ Indeed, the optical contribution to the net torque exerted on the director is, at least, four order of magnitude smaller than the electrical contribution, ensured by having $P / P_{\text {th }} \lesssim 10^{-4} \ll$ $\left(V / V_{\text {th }}\right)^{2} \sim 1$, according to the optical Fréedericksz threshold power estimated from Ref. 27.

${ }^{26}$ R. Barboza, U. Bortolozzo, M. G. Clerc, S. Residori, and E. Vidal-Henriquez, Light-matter interaction induces a single positive vortex with swirling arms, Phil. Trans. R. Soc. London A 372 (20140019).

${ }^{27}$ E. Brasselet, A. Lherbier, and L. J. Dubé, Transverse nonlocal effects in optical reorientation of nematic liquid crystals, J. Opt. Soc. Am. B 23, 36-44 (2006). 